\title{
On the Flow of Polythermal Glaciers. II. Surface Wave Analysis
}

\author{
A. C. Fowler; D. A. Larson \\ Proceedings of the Royal Society of London. Series A, Mathematical and Physical Sciences, Vol. \\ 370, No. 1741. (Mar. 12, 1980), pp. 155-171.
}

Stable URL:

http://links.jstor.org/sici?sici=0080-4630\%2819800312\%29370\%3A1741\%3C155\%3AOTFOPG\%3E2.0.CO\%3B2-G

Proceedings of the Royal Society of London. Series A, Mathematical and Physical Sciences is currently published by The Royal Society.

Your use of the JSTOR archive indicates your acceptance of JSTOR's Terms and Conditions of Use, available at http://www.jstor.org/about/terms.html. JSTOR's Terms and Conditions of Use provides, in part, that unless you have obtained prior permission, you may not download an entire issue of a journal or multiple copies of articles, and you may use content in the JSTOR archive only for your personal, non-commercial use.

Please contact the publisher regarding any further use of this work. Publisher contact information may be obtained at http://www.jstor.org/journals/rsl.html.

Each copy of any part of a JSTOR transmission must contain the same copyright notice that appears on the screen or printed page of such transmission.

The JSTOR Archive is a trusted digital repository providing for long-term preservation and access to leading academic journals and scholarly literature from around the world. The Archive is supported by libraries, scholarly societies, publishers, and foundations. It is an initiative of JSTOR, a not-for-profit organization with a mission to help the scholarly community take advantage of advances in technology. For more information regarding JSTOR, please contact support@jstor.org. 


\title{
On the flow of polythermal glaciers. II. Surface wave analysis $\dagger$
}

\author{
By A. C. Fowler $\ddagger$ AND D. A. Larson§ \\ + School of Mathematics, Trinity College, Dublin, Republic of Ireland \\ $\S$ Mathematics Department, Indiana University, Bloomington \\ Indiana 47401, U.S.A.
}

(Communicated by Sir Charles Frank, F.R.S. - Received 19 June 1978 Revised 6 August 1979)

\begin{abstract}
We show how the 'reduced' model developed in part I of this paper may be used to derive a nonlinear hyperbolic equation which describes the passage of kinematic waves along the surface of a valley glacier. Qualitative descriptions of large-scale snout movements and the formation and evolution of surface shocks are found from this approach, and earlier results of Nye (I960) are reproduced in the limit where surface disturbance amplitudes are 'small'.
\end{abstract}

\section{INTRODUCTION}

The study of kinematic waves on the surface of glaciers is a subject that has received, and continues to receive, a considerable amount of attention from theoreticians interested in the dynamic properties of glacial ice flow. Such waves are observed as undulations of the ice surface which propagate down-glacier at speeds of the order of three to four times the surface ice speed. They are thus somewhat analogous to water waves.

The mechanism of their propagation is well understood, and their properties (wave speed, etc.) have been studied in numerous works. Among the earliest of these, the paper by Finsterwalder ( 1907 ) is of particular importance. He showed that consideration of an integrated form of the equation of conservation of mass determined a nonlinear hyperbolic equation for the glacier depth; this equation could be solved implicitly by using the method of characteristics. Finsterwalder considered also the formation and propagation of shocks. His work was reviewed by Lliboutry $(1964-5)$.

More recently, Nye (I960, I963a,b) and Weertman (1958) formulated similar models, and were able to explain the observed wave speeds. Nye's work in particular, which uses a linearized analysis based on the kinematic wave theory of Lighthill \& Whitham (I955), is extensively referred to, and one aim of this paper is to provide a nonlinear analysis of an analogue of Nye's surface wave equation: in so doing, we

$\dagger$ Part I appeared in Proc. R. Soc. Lond. A 363, 217-242. 
hope to develop a more complete theory of surface wave phenomena. Our work echoes that of Finsterwalder (1907), but we are also able to provide a quantitative analysis of the effects of small perturbations and of seasonal climatic variation. It is worth emphasizing that a more complete understanding of surface waves may be of assistance in indirectly determining the flow law of ice, basal sliding velocities, and so on.

Our aim here will be to develop a rational theory of large scale nonlinear waves, and we thus show in $\S 2$ how a simple analogue of previously studied equations can be derived from the 'reduced' glacier flow model which was presented in part I: for analytical simplicity, we make the assumptions that the ice is completely cold, and the viscosity is independent of the temperature; arguments are presented as to why the equation should be a useful qualitative model for the description of surface waves on polythermal glaciers in general. The resulting dimensionless equation then describes evolution of the ice thickness $H(x, t)$, and is scaled in such a way that $H$ is assumed to have $O(1)$ derivatives in $x$ : we thus concern ourselves with the evolution of an entire glacier profile. Other authors (Lick I970; Hutter 1979) have given nonlinear analyses which, however, are implicitly concerned with surface motions whose wavelength is much shorter than the glacier itself. Such models would not, for example, be relevant to the study of the effects of climatic variations. In $\S 3$, the general solution of our nonlinear equation is given by means of the method of characteristics, and solutions are described in detail for several particular cases of physical interest. First, we discuss the case of an initially present shock such as might physically represent a sudden flux of ice onto the glacier surface owing to the surging of a tributary. We show that the disturbance always reaches the snout in a finite time, and give a formula for the eventual advance of the snout position. Secondly, we discuss the evolution of an initially small perturbation about the (unique) steady state glacier profile. For this case, we show that shocks can form, but only sufficiently near the snout. We develop a method for constructing leading order approximations for the corresponding solutions, and this method is applied, by way of example, to the study of perturbations induced by high frequency seasonal variations in the surface ice accumulation rate. In $\S 6$, we briefly discuss the mathematical difficulties that arise when we introduce into our surface wave equation appropriate diffusional terms that tend to zero with the ice thickness; a comparable situation is discussed by Nye $(1963 b)$ for a linear equation of similar type. The conclusions of the paper are given in $\S 7$.

\section{EqUATIONS AND STEADY STATE PROFILES}

Our study of glacier surface waves is based upon the reduced flow model of part I; we use the same notation as was used there. We focus upon the case of a cold, non-sliding glacier, the viscosity of which is temperature independent. As is discussed below, surface wave phenomena for more general types of polythermal glaciers are 
expected to be qualitatively the same as for this special case. We therefore assume for now that

throughout the glacier and that

$$
T<T_{Q}
$$

$$
\kappa=0 .
$$

In this case (as in $\S 5$ of part I), the boundary value problems in the reduced model of part I for the stream function and the temperature field uncouple, that for the former being

$$
\left.\begin{array}{rl}
\Psi_{\xi \xi} & =\xi^{n}\left[1-\mu\left(H_{x}+h_{x}\right)\right]^{n}, \\
\Psi & =s(x, t) \quad \text { on } \xi=0, \\
\Psi_{\xi} & =0, \Psi=\frac{\partial}{\partial t} \int_{x_{0}(t)}^{x} H(\sigma, t) \mathrm{d} \sigma \quad \text { on } \quad \xi=H(x, t) .
\end{array}\right\}
$$

Two immediate integrations of this system yield

$$
\Psi(x, t)=s(x, t)-\left[1-\mu\left(H_{x}+h_{x}\right)\right]^{n}\left[\left(\frac{H^{n+1}}{n+1}\right) \xi-\frac{\xi^{n+2}}{(n+1)(n+2)}\right],
$$

with $H(x, t)$ determined from

$$
\frac{\partial}{\partial t} \int_{x_{0}(t)}^{x} H(\sigma, t) \mathrm{d} \sigma=\dot{s}(x, t)-\left[1-\mu\left(H_{x}+h_{x}\right)\right]^{n}\left[\frac{H^{n+2}}{n+2}\right] .
$$

Differentiating this last equation with respect to $x$, we find that ice thickness changes in the glaciers we consider here are governed by the following equation:

$$
H_{t}+\left[\left\{1-\mu\left(H_{x}+h_{x}\right)\right\}^{n}\left(\frac{H^{n+2}}{n+2}\right)\right]_{x}=s_{x}(x, t) .
$$

This last equation is easily seen to be a particular case of Lighthill \& Whitham's (1955) 'kinematic wave equation',

$$
H_{t}+Q_{x}=a(x, t) \equiv s_{x}(x, t)
$$

where $Q$, the flux, is assumed to depend on $x, H$ and $H_{x}$ in some prescribed way. Nye's (1960, 1963 $a, b)$ theory of glacier surface waves is based on a linearized version of equation (2.7). In $\S \S 3-5$ here, we develop a nonlinear theory of surface waves based on equation (2.6); this theory is compared to some extent to that of Nye in $\S 5$. Although equation (2.6) has only been derived for cold non-sliding glaciers for which $\kappa=0$, similar ice thickness evolution equations can also be derived for purely temperate glaciers with $R_{1}$ constant. Furthermore, for any glacier that undergoes small fluctuations only about some stable steady state (i.e. for most non-surge glaciers), one may replace $T$ and $w$ in the reduced model equations of part I by the (assumed known) steady state temperature and moisture content solutions and again derive an approximate ice thickness evolution equation of a type similar to equation (2.6). (Further details of this procedure are given by Fowler i 977.) There- 
fore, even though the assumption that $\kappa=0$ is not a completely realistic one (cf. $\S 3$ of part I), we expect that our theory will provide qualitatively valid descriptions of surface wave phenomena for most (non-surge) polythermal glaciers.

From $\S 3$ of part I, it is expected that $\mu \ll 1$ for many glaciers. In $\S \S 3-5$ here, we therefore make the simplifying assumption that

$$
\mu=0 .
$$

The effect of making this approximation is discussed briefly in $\S 6$. Using equation (2.8), we find that equation (2.6) becomes

$$
H_{t}+H^{n+1} H_{x}=s_{x}(x, t) .
$$

Putting $x=x_{0}(t)$ in equation (2.5), we find that a boundary condition for this equation is

$$
\left.H\left(x_{0}(t), t\right)=\begin{array}{ccc}
{\left[(n+2) x_{0}^{\prime}(t)\right]^{1 /(n+1)}} & \text { for } & x_{0}^{\prime}(t)>0 \\
0 & \text { for } & x_{0}^{\prime}(t) \leqslant 0
\end{array}\right\} .
$$

We also prescribe the initial condition

$$
H(x, 0)=A(x) \text { for } \quad x_{0}(0) \leqslant x \leqslant x_{\mathrm{S}}(0),
$$

$A(x)$ being left arbitrary for now. The initial-boundary value problem posed by equations (2.9)-(2.11) is to be solved for non-negative values of $H$ in regions where $x \geqslant x_{0}(t)$ and $t \geqslant 0$. The snout position, $x_{\mathrm{S}}(t)$, is then defined for each $t \geqslant 0$ as the smallest value of $x>x_{0}(t)$ such that $H(x, t)=0$.

Before considering the full time-dependent problem posed by equations (2.9)$-(2.11)$, let us recall from $\S 5$ of part I that there exists a unique steady state solution to the problem for $s(x, t) \equiv s(x)$ (so that $x_{0}(t) \equiv x_{0}$ ). This solution is given by

$$
H_{0}(x)=[(n+2) s(x)]^{1 /(n+2)}, \quad s\left(x_{\mathrm{S}}\right)=0 .
$$

Similar results are given by Lliboutry (1964-5). It may be observed that if (as is physically reasonable) $s^{\prime}$ is finite at $x_{0}$ and $x_{\mathrm{S}}$, then $H_{0}$ has infinite slope at these points. As discussed by Fowler (1977), this occurs because terms of order $\delta^{2}$ were neglected in deriving the 'reduced model' from the 'scaled model' in part I; inclusion of these terms in a local analysis near $x_{0}$ and $x_{\mathrm{S}}$ reveals that $H_{x}$ is in fact finite at these points.

\section{Characteristic SOLUtions aNd Shock FORMAtion}

To simplify our study of solutions to the problem posed by equations (2.9)-(2.11), we shall in this and ensuing sections consider the case where $s(x, t)$ is independent of time. In $\S 5$ we briefly discuss a situation where this restriction is relaxed to some extent. If $s(x, t) \equiv s(x)$, then $x_{0}(t) \equiv x_{0}$ and equations (2.9)-(2.11) become

$$
\left.\begin{array}{c}
H_{t}+H^{n+1} H_{x}=s^{\prime}(x), \\
H\left(x_{0}, t\right)=0, \quad H(x, 0)=A(x) .
\end{array}\right\}
$$


The parametric equation that defines $x_{\mathrm{S}}(t)$ is

$$
H\left(x_{\mathrm{S}}(t), t\right)=0, \quad x_{\mathrm{S}}(t)>x_{0} .
$$

The problem (3.1) may be formally solved by the method of characteristics (cf. Finsterwalder 1907), its characteristic form being

$$
\left.\begin{array}{l}
\mathrm{d} x / \mathrm{d} t=H^{n+1}, \quad \mathrm{~d} H / \mathrm{d} t=s^{\prime}(x), \\
H\left(x_{0}, t\right)=0 \quad \text { for } \quad t \geqslant 0, \\
H=A(\sigma), \quad x=\sigma \quad \text { on } \quad t=0,
\end{array}\right\}
$$

where $\sigma \in\left[x_{0}, x_{\mathrm{S}}(0)\right]$ is a characteristic parameter.

Before solving equations (3.3), let us note from equation (2.4) that the surface velocity $u_{\mathrm{s}}$ of the ice is given by

$$
u_{\mathrm{s}}=-\left.\Psi_{\xi}\right|_{\xi=0}=\left(\frac{H^{n+1}}{n+1}\right) .
$$

Comparing this result with the first equation in (3.3), we see that the wave speed of solutions to (3.3)-i.e. the speed at which ice thickness disturbances are propagated along the glacier - is equal to $(n+1)$ times the surface speed, i.e. $4 u_{\mathrm{s}}$. As mentioned previously, this result was derived by Nye (1960) and is in good agreement with field observation.

To solve equations (3.3), we note immediately that a first integral is given by

$$
H^{n+2} /(n+2)=s(x)-s_{1}(\sigma),
$$

where $s_{1}(\sigma)$ is defined for all $\sigma \in\left[x_{0}, x_{\mathrm{S}}(0)\right]$ by

$$
A^{n+2}(\sigma) /(n+2)=s(\sigma)-s_{1}(\sigma),
$$

and hence represents the change that must be made to the steady state flux function $s(\sigma)$ in order that $A(\sigma)$ be the corresponding steady state ice thickness profile. In what follows, we shall regularly think of $s_{1}(\sigma)$ instead of $A(\sigma)$ as the initial data for equations (3.3); in doing this, it must be remembered that the restrictions placed on $A(\sigma)$ above imply that $s_{1}\left(x_{0}\right)=0, s_{1}\left(x_{\mathrm{S}}(0)\right)=s\left(x_{\mathrm{S}}(0)\right)$ and $s_{1}(\sigma)<s(\sigma)$ for all $\sigma \in\left(x_{0}, x_{\mathrm{S}}(0)\right)$. Substituting equation (3.5) into the first equation in (3.3) and solving the resulting initial value problem, we obtain

$$
t=\int_{\sigma}^{x} \frac{\mathrm{d} x}{\left[(n+2)\left\{s(x)-s_{1}(\sigma)\right\}\right]^{(n+1)(n+2)}},
$$

which describes the $(x, t)$ pairs that collectively comprise the characteristic parametrized by $\sigma$. Along this characteristic, $H(x, t)$ is then found from equation (3.5), and so equations (3.5) and (3.7) provide a formal characteristic solution for equation (3.1). 
To illustrate the general nature of the characteristics defined from equation (3.7), let us consider the special case that corresponds to a steady state ice flow with $x_{\mathrm{S}}(t) \equiv x_{\mathrm{S}}(0)=x_{\mathrm{S}}$. In this case, $s_{1}(\sigma) \equiv 0$, and equation (3.7) becomes

$$
t=\int_{\sigma}^{x} \frac{\mathrm{d} x}{[(n+2) s(x)]^{(n+1) /(n+2)}} .
$$

Along any characteristic (where $\sigma$ is constant), equation (3.8) implies that as $t$ is increased, $x$ increases until $x=x_{\mathrm{S}}$, at which point $H=0$ and the characteristic slope $\mathrm{d} t / \mathrm{d} x=+\infty$. This time taken for an initial disturbance at $x=\sigma$ to propagate along the characteristic and reach this point is then given by equation (3.8) with $x=x_{\mathrm{S}}$. This time is finite or infinite according to whether $[s(x)]^{-(n+1) /(n+2)}$ is integrable or not in a neighbourhood about $x=x_{\mathrm{S}}$. The former case is generally true, since the ablation rate at the snout is typically finite; we assume there that this is indeed the case. A typical steady state characteristic diagram is then as shown in figure 1. (This figure is similar to one given by Nye 1960.)

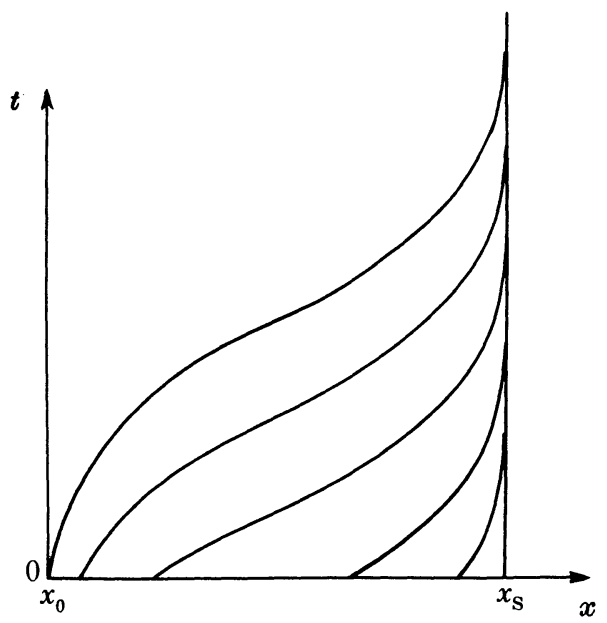

Frgure 1. Typical steady state characteristics.

In the general case where $s_{1}(\sigma) \not \equiv 0$, equations (3.5) and (3.7) implicitly provide the unique solution to equation (3.1) in $(x, t)$-regions where $\sigma(x, t)$ is single-valued. In such regions this solution is qualitatively the same as that for the $s_{1}(\sigma) \equiv 0$ case, characteristics having shapes similar to those shown in figure 1. Along any specific characteristic, $x$ increases with $t$ (cf. figure 1) until an $(x, t)$ point is reached where either $\sigma$ becomes multi-valued (and the solution ceases to be valid) or $s(x)=s_{1}(\sigma)$. At points where this second condition holds we have $H=0$, which marks the glacier snout position, and hence $x=x_{\mathrm{S}}(t)$ and the characteristics are considered to terminate. The time evolution of the snout position $x_{\mathrm{S}}(t)$ is then completely described 
for increasing values of $t \geqslant 0$, as long as $\sigma\left(x_{\mathrm{S}}(t), t\right)$ remains single-valued, by the parametric equations

$$
\left.\begin{array}{c}
s\left(x_{\mathrm{S}}(t)\right)=s_{1}(\sigma), \\
t=\int_{\sigma}^{x_{\mathrm{S}}(t)} \frac{\mathrm{d} x}{\left[(n+2)\left\{s(x)-s_{1}(\sigma)\right\}\right]^{(n+1))((n+2)}} \cdot
\end{array}\right\}
$$

The time taken for an initial disturbance at $x=\sigma$ to propagate along the characteristic parametrized by $\sigma$ and reach the snout, assuming $\sigma$ is single valued along this path, is then given by the second equation in (3.9). We shall again assume that for all relevant $\sigma,\left[s(x)-s_{1}(\sigma)\right]^{-(n+1) /(n+2)}$ is integrable in any $x$-neighbourhood about $x=x_{\mathrm{S}}(t)$, so that this time is finite.

If $\sigma(x, t)$ is uniquely defined from equation (3.7) for all $x \in\left[x_{0}, x_{\mathrm{S}}(t)\right]$ and $t \geqslant 0$, $x_{\mathrm{S}}(t)$ being defined for $t \geqslant 0$ from equation (3.9), then equations (3.5), (3.7) and (3.9) provide the complete solution to equation (3.1). As we shall now show, this is the case if and only if $s_{1}^{\prime}(\sigma) \leqslant 0$ for all $\sigma \in\left[x_{0}, x_{\mathrm{S}}(0)\right]$ : if $s_{1}^{\prime}(\sigma)>0$ for some such $\sigma$, then equation (3.7) does not provide a single-valued prescription of $\sigma(x, t)$ for all relevant $(x, t)$ pairs. The implicit function theorem guarantees that at any $(x, t)$ point where $\sigma$ is multivalued (i.e. where characteristics intersect), both (3.7) and the 'envelope equation',

$$
\frac{\partial}{\partial \sigma}\left[\int_{\sigma}^{x} \frac{\mathrm{d} x}{\left[(n+2)\left\{s(x)-s_{1}(\sigma)\right\}\right]^{(n+1) /(n+2)}}\right]=0,
$$

(i.e. the result of differentiating (3.7) with respect to $\sigma$ ) must be satisfied. Performing the differentiation in equation (3.10) and using equation (3.6), we find that equation (3.10) can be rewritten as

$$
\frac{1}{A^{n+1}(\sigma)}=(n+1) s_{1}^{\prime}(\sigma) \int_{\sigma}^{x} \frac{\mathrm{d} x}{\left.\left[(n+2)\left\{s(x)-s_{1}(\sigma)\right\}\right]^{1+[n+1 / n+2}\right]} .
$$

Since $A(\sigma) \geqslant 0$ for all $\sigma \in\left[x_{0}, x_{\mathrm{S}}(0)\right]$ and the integrand on the right-hand side of equation (3.11) is positive for all relevant $(x, \sigma)$ pairs, it is clear that equation (3.11) can never be satisfied if $s_{1}^{\prime}(\sigma) \leqslant 0$ for all $\sigma \in\left[x_{0}, x_{S}(0)\right]$. Conversely, if $s_{1}^{\prime}(\sigma)>0$ for some such $\sigma$, then the right-hand side of equation (3.11) increases continuously from zero to infinity as the $(x, t)$ characteristic path parametrized by $\sigma$ and defined from equation (3.7) is traversed from the point where $(x, t)=(\sigma, 0)$ to the point where $s(x)=s_{1}(\sigma)$. There clearly must then be some intermediate $(x, t)$ point on this path for which both equation (3.7) and equation (3.11) are satisfied for this value of $\sigma$, and hence, as claimed, the prescription of $\sigma(x, t)$ in equation (3.7) is single-valued for all relevant $(x, t)$ pairs if and only if $s_{1}^{\prime}(\sigma) \leqslant 0$ for all $\sigma \in\left[x_{0}, x_{\mathrm{S}}(0)\right]$.

In general, $s_{1}^{\prime}(\sigma)$ will be positive for some $\sigma \in\left[x_{0}, x_{S}(0)\right]$ and so equations (3.5), (3.7) and (3.9) will provide a unique solution for equation (3.1) only for values of $t \in\left[0, t_{\mathrm{c}}\right), t_{\mathrm{c}}$ being the infimum of all positive times $t$ for which $\sigma(x, t)$ is not singlevalued for all $x \in\left[x_{0}, x_{S}(0)\right]$. Standard 'shock' theory for hyperbolic conservation laws (see, for example, Murray 1970) can be used, however, to provide a discon- 
tinuous but physically meaningful extension of this solution for times $t \geqslant t_{\mathrm{c}}$. Along any path in the $(x, t)$ plane where a discontinuity in the solution (i.e. a shock) occurs, we require that the solution represent the physical fact that mass must be conserved across the path. If we denote the position of any specific shock at time $t$ by $x_{\mathrm{d}}(t)$, then in the usual way (Murray 1970) the velocity of the shock is given by

$$
\frac{\mathrm{d} x_{\mathrm{d}}}{\mathrm{d} t}=\left[\frac{H^{n+2}\left(x_{\mathrm{d}}^{-}(t), t\right)-H^{n+2}\left(x_{\mathrm{d}}^{+}(t), t\right)}{(n+2)\left[H\left(x_{\mathrm{d}}^{-}(t)\right)-H\left(x_{\mathrm{d}}^{+}(t), t\right)\right]}\right] .
$$

(see also Fowler 1977). Away from shocks, the extended solution is given by equations (3.5) and (3.7). In order that this solution be uniquely defined for all $t \geqslant t_{c}$, we must consider any characteristic defined by equation (3.7) to be terminated at any $(x, t)$ point where it intersects a shock. From equation (3.7), $x$ increases as $t$ increases along any such characteristic and, assuming the characteristic emanating from $x=x_{0}$ at $t=0$ does not intersect any shock before it terminates at the snout position $x_{\mathrm{S}}\left(t_{\mathrm{F}}\right)$ for some time $t_{\mathrm{F}}>0$, we find that $t_{\mathrm{F}}$ and $x_{\mathrm{S}}\left(t_{\mathrm{F}}\right)$ are given explicitly from equation (3.9) and the fact that $s_{1}\left(x_{0}\right)=0$ by

$$
t_{\mathrm{F}}=\int_{x_{0}}^{x_{\mathrm{S}}\left(t_{\mathrm{F}}\right)} \frac{\mathrm{d} x}{[(n+2) s(x)]^{(n+1) /(n+2)}}, \quad s\left(x_{\mathrm{S}}\left(t_{\mathrm{F}}\right)\right)=0 .
$$

From the assumptions made above, $t_{\mathrm{F}}$ is finite; by this time, all initial disturbances have reached the glacier snout, and the ice flow is steady for all subsequent times.

In general, the extended solution to equation (3.1) for $t \geqslant t_{\mathrm{c}}$ will involve multiple shocks and must be studied on a case-by-case basis. In the next two sections, we indicate the flavour of such analyses by considering several special cases of (3.1) which we feel are of intrinsic interest in their own right.

\section{Evolution OF AN INITIALLY PRESENT SHOCK}

In order to illustrate in a more detailed way the nature of characteristic solutions to equation (3.1) when shocks are present, we consider equation (3.1) here with a special restriction on the initial data. Specifically, we consider the case where for some $\left(\sigma_{1}, \sigma_{2}\right) \subset\left[x_{0}, x_{\mathrm{S}}(0)\right]$ and $c>0$,

$$
\left.s_{1}(\sigma)=\begin{array}{ll}
-c & \text { for } \sigma_{1}<\sigma<\sigma_{2} \\
0 & \text { otherwise }
\end{array}\right\}
$$

so that shocks are initially present at $x=\sigma_{1}$ and $x=\sigma_{2}$ as illustrated in figure 2 . This initial information models, for example, the physical situation existing after the surge of a tributary glacier has deposited a large quantity of material on the main glacier surface.

A typical characteristic diagram for this first special case is displayed in figure 3. In constructing this diagrąm, we have argued as follows. It is clear that a single 


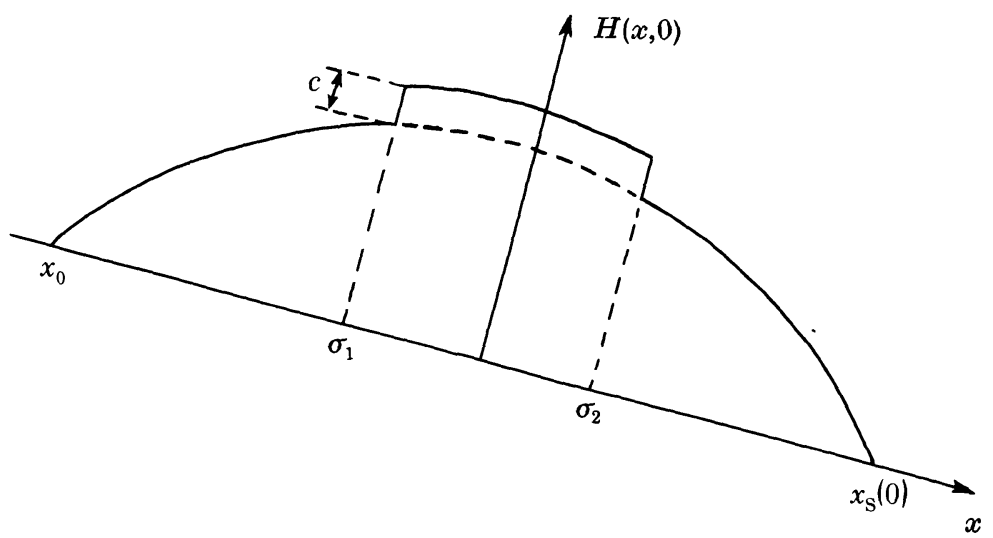

Figure 2. The initial glacier profile with shocks.

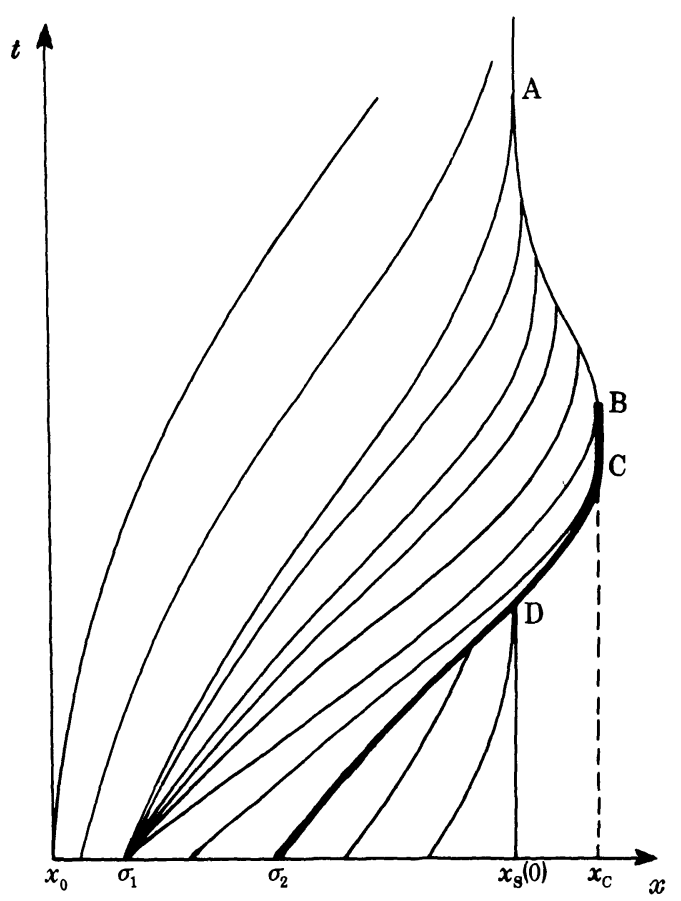

Figure 3. Characteristic diagram for the case of an initially present shock.

shock must emanate from $\sigma_{2}$ : this will be denoted by $\sigma_{2} \mathrm{C}, \mathrm{C}$ being the point where the shock terminates (where the jump in $H$ has decayed to zero). It is also mathematically possible (as shown by Fowler I977) that a second shock emanates from $\sigma_{1}$, but, as discussed by Whitham (1974, p. 38), such a shock is structurally unstable and hence physically unrealistic. On the other hand, there exists a perfectly good continuous single-valued solution available for the characteristics emanating from the vicinity of $\sigma_{1}$ : as shown in figure 3 , the characteristic diagram for this solution takes the form of an expansion fan. On physical grounds, we therefore consider only 
this possible solution in what follows. The characteristics that begin on $\sigma_{2} x_{\mathrm{S}}(0)$ and terminate on either $\sigma_{2} \mathrm{D}+$ (i.e. the right-hand side of this shock) or $x_{\mathrm{S}}(0) \mathrm{D}$ are 'steady state' ones as given in equation (3.8); this is also the case for the ones beginning on $x_{0} \sigma_{1}$ and terminating on the line $x=x_{\mathrm{S}}(0)$. We denote by $\sigma_{1} \mathrm{~A}$ the 'lowest' such steady state characteristic, A being the point where this characteristic reaches the snout.

If we suppose that $\mathrm{C}$ lies in $x \leqslant x_{\mathrm{s}}(0)$, then at this point $\sigma_{2} \mathrm{C}$ must be tangential to two characteristics, one coming from $\sigma_{2} x_{\mathrm{S}}(0)$ and the other from $\sigma_{1} \sigma_{2}$ or $x_{0} \sigma_{1}$. Along the first one, $H^{n+2} /(n+2)=s(x)$, and since $H$ is continuous at $\mathrm{C}$ (because the shock terminates there), this must also be true along the second one, which is therefore also a steady state characteristic, and thus can only emanate from $x_{0} \sigma_{1}$. However, distinct steady state characteristics can never intersect, and so we are forced to conclude that $\mathrm{C}$ can actually lie only in $x>x_{\mathrm{S}}(0)$. Initially present shocks of the type considered here must therefore always lead to a temporary advance of the snout.

We denote by $\mathrm{D}$ the point where $\sigma_{2} \mathrm{C}$ and $x=x_{\mathrm{S}}(0)$ intersect. From equation (3.12), DC is described by

$$
\frac{\mathrm{d} x_{\mathrm{S}}}{\mathrm{d} t}=\frac{H^{n+1}\left(x_{\mathrm{S}}^{-}(t), t\right)}{n+2}
$$

On comparing equation (4.2) with equation (3.4), we see that (when there is a shock at the snout) the rate of advance of the snout is equal to $(n+1) /(n+2) \approx \frac{4}{5}$ times the local surface speed. This prediction should be capable of field verification, unless altered by the effects of 'diffusion' (see $\S 6$ ) and seasonal variations.

DC terminates (at $\mathrm{C}$ ) when the jump in $H$-which is $H\left(x_{\mathrm{S}}^{-}(t), t\right)$ here-becomes zero. From equation (4.2), $\mathrm{d} x_{\mathrm{S}} / \mathrm{d} t=0$ there, so that $\sigma_{2} \mathrm{C}$ is tangential at $\mathrm{C}$ to a vertical line. We denote by $\mathrm{B}$ the point where the characteristic emanating from $\sigma_{1}+$ meets $\sigma_{2}$ DCA (the DCA part of this curve being the $x=x_{\mathrm{S}}(t)$ snout curve). If, as portrayed in figure $3, \mathrm{~B}$ lies on $\mathrm{CA}$, then all characteristics arriving at $\mathrm{CB}$ emanate from $\sigma_{1} \sigma_{2}$ and the equations in (3.5), (3.7) and (3.9) completely describe the solution of equation (3.1) along these characteristics. In particular, since $s_{1}(\sigma) \equiv-c$ for $\sigma \in\left(\sigma_{1}, \sigma_{2}\right)$, the first equation in (3.9) shows that (as portrayed in figure 3) CB is vertical. On the other hand, characteristics arriving at $\mathrm{BA}$ emanate from the expansion fan at $\sigma_{1}$, within which $\left[H^{n+2} /(n+2)-s(x)\right]$ decreases from $c$ (on $\sigma_{1} \mathrm{~B}$ ) to 0 (on $\sigma_{1} \mathrm{~A}$ ). If we parametrize the characteristics within the expansion fan at $\sigma_{1}$ by the value $\tilde{s}_{1},-c \leqslant \tilde{s}_{1} \leqslant 0$, so that the $\tilde{s}_{1}$ characteristic is the curve along which

$$
H^{n+2} /(n+2)=s(x)-\tilde{s}_{1},
$$

then the equations in (3.5), (3.7) and (3.9) with $\sigma$ and $s_{1}$ replaced by $\sigma_{1}$ and $\tilde{s}_{1}$ completely describe the solution of equation (3.1) along these characteristics. In particular, since $\tilde{s}_{1}$ increases monotonically as the expansion fan is traversed in an anticlockwise manner, and $s$ is assumed to be monotonically decreasing in $x$ for $x \geqslant x_{\mathrm{S}}(0)$, the first equation in (3.9) now shows that $x_{\mathrm{S}}(t)$ is monotonically 
decreasing in $t$ as $\mathrm{BA}$ is traversed. It then follows from equation (4.2) that $x_{\mathrm{S}}$ is monotonically increasing as $\mathrm{DC}$ is traversed and hence, as shown in figure $3, \mathrm{~B}$ must indeed be on CA. The point $\mathrm{C}$ therefore marks the maximum advance of the snout, and it is of interest to compute what this advance is. Along any characteristic arriving at $\sigma_{2} \mathrm{DC}-, H^{n+2} /(n+2)=s(x)+c$. Since $H$ must equal zero at $\mathrm{C}$, if we denote the solution of $s(x)+c=0$ by $x_{\mathrm{C}}$, then

$$
x=x_{\mathrm{C}} \text { at } \mathrm{C},
$$

and $x_{\mathrm{C}}$ marks the maximum advance of the snout. If $c$ is small, then we may write $s\left(x_{\mathrm{C}}\right) \approx-a_{\mathrm{S}}\left(x_{\mathrm{C}}-x_{\mathrm{S}}(0)\right)$, where $a_{\mathrm{S}}=-s^{\prime}\left(x_{\mathrm{S}}(0)\right)$ is the ice ablation rate at the steady state snout, and hence we have

$$
x_{\mathrm{C}}-x_{\mathrm{S}}(0) \approx c / a_{\mathrm{S}} .
$$

Denoting dimensional variables by a superscript $\mathrm{D}$, we then have that the maximum advance $\Delta x^{\mathrm{D}}$ of the snout past its steady state position is estimated by

$$
\Delta x^{\mathrm{D}} \approx\left(c^{\mathrm{D}} / a_{\mathrm{S}}^{\mathrm{D}}\right)(l V / d)=U\left(c^{\mathrm{D}} / a_{\mathrm{S}}^{\mathrm{D}}\right),
$$

where $U$ is a typical longitudinal ice velocity, and $d, l$ and $V$ are as defined in part I of this paper. If, for example, $U \approx 100 \mathrm{~m} \mathrm{a}^{-1}, a_{\mathrm{S}}^{\mathrm{D}} \approx 5 \mathrm{~m} \mathrm{a}^{-1}$ and $c^{\mathrm{D}} \approx 10 \mathrm{~m}$ of ice is suddenly applied to some region of the glacier surface, then the snout will eventually advance a distance $\Delta x^{\mathrm{D}} \approx 200 \mathrm{~m}$ before retreating. It is seen from equation (4.5) that the maximum advance is independent of the length $\sigma_{2}-\sigma_{1}$ of the initial disturbance. This length clearly plays an important role, however, in determining the length of $\mathrm{CB}$ : the larger $\sigma_{2}-\sigma_{1}$ is the longer the snout will dwell at its position of maximum advance before retreating. In this case, an upper bound $\bar{t}_{\mathrm{CB}}$ on the (non-dimensional) time period during which the snout remains at this position can be given from equations (3.9) and (4.1) by

$$
\bar{t}_{\mathrm{CB}}=\int_{\sigma_{2}}^{\sigma_{1}} \frac{\mathrm{d} x}{[(n+2)\{s(x)+c\}]^{(n+1) /(n+2)}},
$$

this being dimensionally on the order of the decay time for the disturbance.

\section{Approximate results for small disturbances}

In this section, we discuss further the characteristic solution of the surface wave problem in two distinct cases where the solution represents a small perturbation about the steady state solution (2.12). First, we consider the effect of initially imposing a small perturbation on the steady state ice profile. Secondly, we discuss the nature of ice thickness perturbations induced by high frequency seasonal variations in the surface ice accumulation rate.

We suppose first that $s(x, t) \equiv s(x)$; in this case the solution of the surface wave problem is as described in general in $\S 3$. We define

$$
\Sigma=\max _{\sigma \in\left[x_{0}, x_{\mathrm{s}}(0)\right]}\left\{\left|s_{1}^{\prime}(\sigma)\right|\right\}
$$


and assume that

$$
\Sigma \ll 1
$$

so that we consider here the limit where $\Sigma$ tends towards zero; it then follows from equation (5.1) that

$$
\max _{\sigma \in\left[x_{0}, x_{s}(0)\right]}\left\{\left|s_{1}(\sigma)\right|\right\}=O(\Sigma)
$$

as $\Sigma \rightarrow 0$. From equations (3.5) and (3.7), the general solution away from shocks is given by

$$
\left.\begin{array}{c}
H^{n+2} /(n+2)=s(x)-s_{1}(\sigma), \\
t=\int_{\sigma}^{x} \frac{\mathrm{d} x}{\left[(n+2)\left\{s(x)-s_{1}(\sigma)\right\}\right]^{(n+1) /(n+2)}},
\end{array}\right\}
$$

and if a shock forms at some point $(x, t)$ along the characteristic parametrized by $\sigma$, then from equation (3.11), $x=X(\sigma)$ must satisfy

$$
\frac{1}{A^{n+1}(\sigma)}=(n+1) s_{1}^{\prime}(\sigma) \int_{\sigma}^{X(\sigma)} \frac{\mathrm{d} x}{\left[(n+2)\left\{s(x)-s_{1}(\sigma)\right\}\right]^{1+(n+1) /(n+2)}} .
$$

As $\Sigma \rightarrow 0, A^{n+1}(\sigma)$ is uniformly $O(1)$ while $s_{1}^{\prime}(\sigma)$ is uniformly $O(\Sigma)$, and hence it is clear that this condition (5.4) can be met for any fixed value of $\sigma \in\left(x_{0}, x_{\mathrm{S}}(0)\right)$ only if $s(X(\sigma))-s_{1}(\sigma)=o(1)$-i.e. $X(\sigma)$ is sufficiently near the $x$ value at which the integrand in equation (5.4) is singular. Since it has been shown in $\S 3$ that the equation $s(x)-s_{1}(\sigma)=0$ formally defines the snout or the head of the glacier, it then follows that shocks can form only very near the snout (or, less interestingly, the head) of the glacier for $\Sigma \ll 1$.

We now wish to develop approximate expressions for the general solution given in equation (5.3) under the assumption that $\Sigma \ll 1$. The first work of this type was done by Nye (1960), who sought approximate solutions to a linearized version of equation (2.7) under this assumption. The result of his analysis is that small depth perturbations eventually decay to zero, but not uniformly in $x$, as may be seen from figure 4 (after Nye). In Nye's words, 'the lower parts thicken unstably until a kinematic wave arrives to restore stability'. This non-uniformity in the approximation arises from the linearization of equation (2.7) about a steady state depth profile which goes to zero as $x \rightarrow x_{\mathrm{S}}(0)$. This difficulty is avoided in the following analysis where the approximate solution we develop from equation (5.3) is uniformly valid over its domain of definition, which is upstream from shocks. We have already shown that such shocks can only exist near the snout, and so our approximate solution is uniformly valid over most of the glacier. To complete the approximation, we could use the shock analysis presented in $\S 3$, but in view of the comments on diffusion effects to be given in $\S 6$ and those on model rescalings in the snout region presented by Fowler (1977), this is hardly a useful exercise. In any case, it is clear (since the depth decreases at such shocks) that the solution so extended would not grow in time in the manner of Nye's solution, and in this sense our approximate solution is considered to be uniformly valid in $x$. 


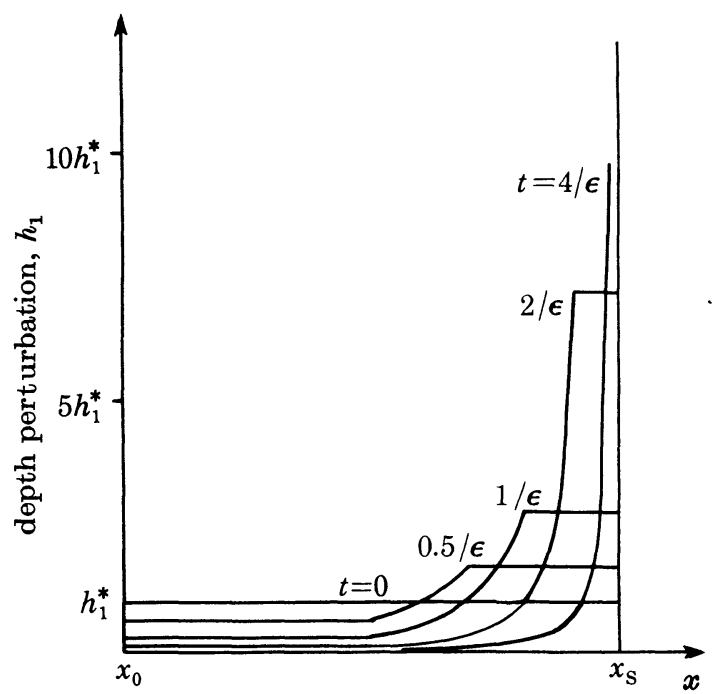

Figure 4. Typical time evolution of initially small depth perturbations as predicted by Nye ( 1960$)(1 / \epsilon \equiv$ basic unit of time).

We assume here, as we did in $\S 3$, that $[s(x)]^{-(n+1) /(n+2)}$ is integrable in any neighbourhood about the point where this expression is singular; as discussed there, this assumption is felt to be generally justified. As $\Sigma \rightarrow 0$, the second equation in (5.3) may then be expanded (upstream of any shocks) as

so that

$$
t=\int_{\sigma}^{x} \frac{\mathrm{d} x}{[(n+2) s(x)]^{(n+1) /(n+2)}}+o(1)
$$

with $\sigma_{0}(x, t)$ defined from

$$
\sigma=\sigma_{0}(x, t)+o(1)
$$

$$
t=\int_{\sigma_{0}}^{x} \frac{\mathrm{d} x}{[(n+2) s(x)]^{(n+1) /(n+2)}}
$$

and hence from the first equation in (5.3),

$$
H^{n+2} /(n+2)=s(x)-s_{1}\left(\sigma_{0}\right)+o(\Sigma) .
$$

We immediately see, on neglecting $o(\Sigma)$ in equation (5.8) and using the definition of $\sigma_{0}$ from equation (5.7), that the first-order approximation to the perturbed depth $H(x, t)$ is then given by the solution of the problem

$$
\left.\begin{array}{c}
H_{t}+H_{0}^{n+1} H_{x}=s^{\prime}(x), \\
H=A\left(\sigma_{0}\right), \quad x=\sigma_{0} \quad \text { on } \quad t=0,
\end{array}\right\}
$$

where $H_{0}(x)$ is the steady state solution defined by

$$
H_{0}^{n+2} /(n+2)=s(x) .
$$


Letting

$$
\xi=\int_{x_{0}}^{x} \frac{\mathrm{d} x}{H_{0}^{n+1}}, \quad f(\xi) \equiv H_{0}(x),
$$

we find that the first equation in (5.9) becomes

$$
H_{t}+H_{\xi}=f^{\prime}(\xi)
$$

Subject to the second equation in (5.9), equation (5.12) has the solution

where

$$
H=f(\xi)+\phi(\xi-t)=H_{0}(x)+\phi(\xi-t)
$$

$$
\phi(\xi)=A(x)-H_{0}(x)
$$

is the initial perturbation in $H$. The first-order correction term for the depth profile is thus $\phi(\xi-t)$; this term is uniformly bounded and valid upstream from shocks as $\Sigma \rightarrow 0$ and illustrates very clearly the travelling wave nature of the evolution of ice depth perturbations.

In all sections so far, we have for simplicity assumed that the flux function $s(x, t)$ is independent of time. We now demonstrate that ice depth profiles corresponding to fluxes $s(x, t)$ and $s(x)$ evolve very similarly in time if $s(x)$ is the time average of $s(x, t)$ and $s(x, t)$ oscillates very rapidly in time. For example, this means that seasonal flux variations have a negligible effect on the longer term evolution of ice depth profiles. Instead of equation (3.1), we consider here the model problem

$$
H_{t}+H^{n+1} H_{x}=s^{\prime}(x)+s_{1}^{\prime}(x) \mathrm{e}^{\mathrm{i} \omega t}, \quad H\left(x_{0}, t\right)=0,
$$

where $s_{1}^{\prime}\left(x_{0}\right)=0$ (in order that the problem be consistently posed at $x=x_{0}$ ), $H_{0}$ is as defined in equation (5.10), $\omega \gg 1$ and the initial data are left unspecified, but is restricted to be everywhere within $O(1 / \omega)$ of the steady state $H_{0}$ as $1 / \omega \rightarrow 0$.

A slight modification of the analysis used above to derive equation (5.8) from equation (5.1) then shows that

$$
H=H_{0}+O(1 / \omega)
$$

as $1 / \omega \rightarrow 0$, i.e. as claimed, the solution of equation (5.15) is very similar to that for the case of $s_{1}^{\prime}(x) \equiv 0$ as long as $\omega \gg 1$. We can now use equation (5.16) to determine the nature of this $O(1 / \omega)$ term.

As was true for the first small disturbance problem considered earlier in this section, the characteristics for equation (5.15) are then described in the $1 / \omega \rightarrow 0$ limit by equations (5.6) and (5.7), at least upstream from possible shock regions near the snout. Substituting equation (5.6) into the solution of equation (5.15) and neglecting $o(1 / \omega)$, one then finds that $H(x, t)$ is given by an equation involving the parameter $\sigma_{0}$ defined by equation (5.7): on eliminating $\sigma_{0}$ one obtains the following approximate equation for $H$, which on comparison with equation (5.15) is seen to be directly obtainable from that equation by a straightforward linearization of the characteristics:

$$
H_{t}+H_{0}^{n+1} H_{x}=s^{\prime}(x)+s_{1}^{\prime}(x) \mathrm{e}^{\mathrm{i} \omega t}, \quad H\left(x_{0}, t\right)=0 .
$$


The error in determining $H$ from this equation is $o(1 / \omega)$. Using the variable changes

and defining

$$
\xi=\int_{x_{0}}^{x} \frac{\mathrm{d} x}{H_{0}^{n+1}(x)}, \quad f_{1}(\xi) \equiv s_{1}^{\prime}(x),
$$

$$
H=H_{0}+\phi(\xi, t), \quad \phi=O(1 / \omega) \quad \text { as } \quad 1 / \omega \rightarrow 0,
$$

we find that $\phi$ satisfies

$$
\phi_{t}+\phi_{\xi}=f_{1}(\xi) \mathrm{e}^{\mathrm{i} \omega t},
$$

of which the solution satisfying $\phi(0, t)=0$ is

$$
\begin{aligned}
\phi & =\phi_{0}(\xi-t)+\mathrm{e}^{\mathrm{i} \omega t} \int_{0}^{\xi} e^{\mathrm{i} \omega(z-\xi)} f_{1}(z) \mathrm{d} z \\
& =\phi_{0}(\xi-t)+\frac{1}{i \omega} f_{1}(\xi) \mathrm{e}^{\mathrm{i} \omega t}+O\left(\frac{1}{\omega^{2}}\right),
\end{aligned}
$$

where we have used $f_{1}(0)=0 . \phi_{0}$ is determined from the initial conditions, and by assumption is $O(1 / \omega)$. We see that the effect of a non-zero $\phi_{0}$ is a transient one which vanishes in finite time. After this time, the depth $H$ is described from equations (5.19) and (5.21) by

$$
H(x, t)=H_{0}(x)+\frac{1}{\mathrm{i} \omega} s_{1}^{\prime}(x) \mathrm{e}^{\mathrm{i} \omega t}+o\left(\frac{1}{\omega}\right),
$$

which shows that for $\omega \gg 1$ the leading order deviation of the depth from its steady state profile is simply a time harmonic oscillation of frequency $\omega$ (and amplitude $\left.s_{1}^{\prime}(x)\right)$.

\section{EFFECTS OF DIFFUSION}

In $\S \S 3-5$, we have discussed the manner in which ice thickness profiles change in time by studying the solutions of appropriate initial boundary value problems for equation (2.6) in the physically realistic limit where $\mu=0$. Frequently these solutions have been found to contain discontinuities, i.e. shocks. In models for other physical systems (for example, gas dynamics), where shocks arise when a small parameter multiplying the highest order derivative term in the model equation is set equal to zero, the shock discontinuities are 'smoothed out' in a diffusive manner if the small parameter is made non-zero and of an appropriate sign. Since equation (2.6) can be rewritten in expanded form as

$$
\begin{aligned}
H_{t}+\left[\left\{1-\mu\left(H_{x}+h_{x}\right)\right\}^{n} H^{n+1}\right] H_{x} \\
=\mu\left[(n /(n+2))\left\{1-\mu\left(H_{x}+h_{x}\right)\right\}^{n-1} H^{n+2}\right]\left[H_{x x}+h_{x x}\right]+s_{x}(x, t),
\end{aligned}
$$

and the diffusion coefficient $\mu\left[(n /(n+2))\left\{1-\mu\left(H_{x}+h_{x}\right)\right\}^{n+1} H^{n+2}\right]$ must be positive for almost all relevant $(x, t)$ pairs when $\mu>0$, we therefore expect that the shock solutions found above for equation (2.6) should in fact be smoothed out and made continuous. This may not be true, however, at the snout (and/or head) of the glacier where $H$ and hence the diffusion coefficient are zero. In this section, we 
discuss the difficulties inherent in trying to determine whether or not diffusive effects smooth out shocks which form at the snout (cf $\S 3$ ) and in trying to describe the behaviour of $H(x, t)$ in general for values of $x$ near the snout (or head) when $\mu>0$.

As pointed out in $\S 2$ above, the fact that $H_{x}$ in our analysis is infinite at the snout is inconsistent with the reduction of the scaled model to the reduced model of part I, and hence it may be inappropriate to use any version of equation (6.1) in trying to describe the behaviour of $H(x, t)$ for values of $x$ near the snout position. Based on Fowler's (1977) comparison of the scaled and reduced models we conjecture, however, that this behaviour will in fact be reasonably accurately described by the solution of a properly posed initial boundary value problem for equation (6.1) and the role of additional scaled model terms is simply to make $H_{x}$ finite (but still large in magnitude) at the snout position. If this is true, it is however still not clear as to what constitutes a proper set of spatial boundary conditions for equation (6.1). As noted by Nye ( $1963 b)$, moving boundary problems for parabolic equations such as equation (6.1) typically involve the prescription of three spatial boundary conditions (i.e. two to augment an equation which has second order spatial derivatives and one to describe the moving boundary) and there seem to be only two natural ones, namely equations (2.10) and (3.2), for the physical situation being considered. It is, however, known (see, for example, Smirnova 1963) that many boundary value problems for parabolic equations with diffusion coefficients which degenerate (i.e. become zero) on part of the boundary are properly posed and admit unique solutions only if less than the 'usual' number of boundary data are prescribed. We conjecture that this is indeed the situation for equation (6.1); once equations (3.10), (3.2) and initial data given in (2.11) are prescribed, the resulting moving boundary problem for equation (6.1) admits a unique solution for all $t \geqslant 0$.

\section{CONCLUSIONS}

In this paper, we have shown how a rational model for the motion of twodimensional glacial ice flows, proposed in part I of the paper, may be used to consider the well-known phenomenon of kinematic surface waves. We show how an equation analogous to that considered by Nye (1960) may be analytically derived in a particular asymptotic limit of the reduced model, in which the dependence of the viscosity on temperature and the variation of the surface slope from the mean bedrock slope are neglected (i.e. $\kappa=\mu=0$ ), and also the glacier is assumed not to slide on its base. These assumptions greatly simplify the analysis, but are not expected to alter the results qualitatively. The main result of Nye, namely that the wave speed of disturbances is about four times the surface speed, is of course reproduced, but a nonlinear analysis indicates that shocks (i.e. surface discontinuities) will in general form, and portions of Nye's theory are invalid in such circumstances.

To illustrate the utility of our approach, we use it to study the time evolution of ice depth profiles that result from two particular initial depth profiles of interest. 
First, we consider the case of an initially present shock, such as might represent the result of a surge of a tributary glacier. Using the method of characteristics, we find that such an initial profile inevitably leads to a temporary advance of the snout, and an estimate of the magnitude of this advance is given. Secondly, we consider the effect of a small initial perturbation to the steady state surface profiles (or equivalently, to the steady state surface flux function). In general, shocks will still form, but only sufficiently near the snout; away from this region, we show that there is a uniformly small perturbation to the steady solution which may be explicitly found from the characteristic equations by linearizing the equation for the characteristics (and not the one for the profile itself). The perturbation represents a travelling wave that reaches the snout region in a finite time. Although the solution is not valid all the way to the snout tip, previously described shock arguments could be used to extend it. It is pointed out, however, that the reduced model (though not inconsistent) is not strictly valid near the snout, and it is therefore pointless to consider shocks which form in the snout anyway.

Note. In part I, we referred to certain results on the thermal nature of glacier flow that were to be presented in this paper: this work will now be published elsewhere.

\section{REFERENCES}

Finsterwalder, S. 1907 Z. Gletscherkunde 2, 81.

Fowler, A. C. 1977 Glacier dynamics. D. Phil. thesis, Oxford University.

Fowler, A. C. \& Larson, D. A. 1978 Proc. R. Soc. Lond. A 363, 217.

Hutter, K. 1979 Time dependent surface elevation of an ice slope. J. Glaciol. to appear.

Lick, W. 1970 J. geophys. Res. 75, 2189.

Lighthill, M. J. \& Whitham, G. B. 1955 On kinematic waves. Proc. R. Soc. Lond. A 229, 281.

Lliboutry, L. 1964-5. Traité de Glaciologie, 2 tomes. Paris: Masson et Cie.

Murray, J. D. I970 SIAM J.appl. Math. 19, 273.

Nye, J. F. I 960 Proc. $R$. Soc. Lond. A 256, 559.

Nye, J. F. 1963 a Proc. $R$. Soc. Lond. A 275, 87.

Nye, J. F. $1963 b$ Geophys. J. 7, 431.

Smirnova, G. N. 1963 Sibirsk Mat. Ž. 4, 343 (in Russian).

Weertman, J. I958 IUGG symposium, Chamonix, France, pp. 162-168.

Whitham, G. B. 1974 Linear and nonlinear waves. New York: Wiley-Interscience. 